\title{
Bioassay of Different Agrochemicals and Fertilizers against Root rot (Macrophomina phaseolina) of Soybean in in vitro
}

\author{
B. M. Ingole ${ }^{1^{*}}$ and S. B. Zade ${ }^{2}$ \\ ${ }^{1}$ Department of Plant Pathology Junagadh Agricultural University Junagadh, India \\ ${ }^{2}$ Department of Plant Pathology, Dr. Panjabrao Deshmukh Krishi Vidypeeth, Akola, India \\ *Corresponding author
}

\section{Keywords}

Soybean, Macrophomina phaseolina, Root rot, Fertilizers, Agrochemicals

Article Info

Accepted:

07 March 2020

Available Online:

10 April 2020

\begin{abstract}
A B S T R A C T
Root rot of soybean is an important disease of soybean inflicting heavy losses in crop. The present investigation was carried out to test the efficacy of agrochemicals and fertilizers in vitro. In bioassay test of non systemic fungicides, Zineb $75 \mathrm{WP}$ gave cent per cent inhibition of growth and sclerotial formation at minimum concentration of $500 \mathrm{ppm}$ followed by copper hydroxide 77WP (53.63\%). In systemic fungicides, Carbendazim 50 WP proved best with mean inhibition of 97.81 per cent and completely inhibited the growth of pathogen at higher concentration of $500 \mathrm{ppm}$ followed by Thiophanate methyl 70 WP $(84.57 \%)$ and Tebuconazole 25.9 EC $(80.50 \%)$. They do not allow sclerotial formation at all concentrations tested. The combinations of fungicides Pyraclostrobin 13.3\% WP + Epoxyconazole 5\% WP and Metalaxyl 8\% + Mancozeb 64\% WP gave cent per cent inhibition of mycelial growth and sclerotial formation at all their concentration tested. Among the different herbicides, Quizalophop-p-ethyle 5 EC gave cent per cent inhibition of mycelial growth and sclerotial formation at all concentration followed by the Propaquizafop 10 EC(96.05\%), Oxyfluorfen 23.5EC(88.70\%) and Oxadiargyl $6 \mathrm{EC}(82.23 \%)$. In in vitro testing of chemical fertilizers, Diammonium Phosphate was quite effective in inhibiting growth and sclerotial formation at all concentration followed by Ammonium Sulphate (72.20\%).
\end{abstract}

\section{Introduction}

Soybean (Glycine max (L.) Merill) is also known as golden bean belongs to the family leguminaceae is a very important oil seed crop. It contains about 20 per cent oil and 40 per cent proteins and also the rich source of amino acids, calcium and iron. It is grown in USA, Brazil, China, Russia and India. It is ranks fourth in production of oil seed crops and cultivated in Madhya Pradesh,
Maharashtra, Andhra Pradesh, Rajasthan, U.P and Gujarat. Soybean cultivation has taken a big stride in the country during the past few years. The estimates of world soybean area, production and productivity for 2017-18 are 126.64 million ha, 346.31 million tons and $2735 \mathrm{~kg} / \mathrm{ha}$ and in India soybean grown under area of 10.60 million hectares, production of 8.50 metric tons and average productivity of $802 \mathrm{~kg} / \mathrm{ha}$ during 2017-18. In Maharashtra total area under soybean is about 34.5 lakh 
hectares, production of 2.90 million tons and the average productivity of $841 \mathrm{~kg} / \mathrm{ha}$ during 2017-18 (Anonymous, 2017).

Soybean crop suffers from various foliar and soil borne fungal diseases viz., collar rot, root rot, and charcoal rot (Macrophomina phaseolina), anthracnose (Colletotrichum sp.), bacterial diseases viz., bacterial wilt (Corynebacterium flaccumfaciens), bacterial blight of soybean (Pseudomonas savastanoi pv. glycina), viral disease viz., soybean mosaic (Soybean mosaic virus), soybean leaf curl (Pea streak virus) and nematode disease viz., root knot (Meloidogyne javanica), malady soybean yellow dwarf (Heterodera glycines) (Zafar Iqbal 2012). Yield losses 3050 per cent due to Macrophomina phaseolina in soybean crop has been reported by Yang and Navi, (2005). Losses of plant population up to 77 per cent reported in soybean due to Rhizoctonia bataticola (Muthusamy and Mariappan, 1991). Macrophomina phaseolina causes the root rot in the various regions of the country particularly in northern India. Since last few years, root rot and collar rot disease is being reported in severe proportions from many places.

\section{Materials and Methods}

\section{Isolation}

The fresh collected soybean plants having the disease symptoms of ashy stem blight and shredded bark of the root were used for isolation. The disease causing fungus was isolated by using tissue segment method. Small pieces of tissue from infected stem and root $(3 \mathrm{~mm})$ along with some healthy tissues were cut using sterilized scalpel and the cut tissues were surface sterilized with $0.1 \%$ mercuric chloride for 30 second. The pieces of tissues were subsequently washed in three changes of sterile water and transferred on to Petri dishes containing potato dextrose agar
(PDA) medium before incubating at $28 \pm 1{ }^{0} \mathrm{C}$. The cultures were purified by hyphal tip method and maintained on PDA by storing it under refrigeration $\left(10^{\circ} \mathrm{C}\right)$ and making periodical transfers every month for further study. The fungus was isolated, purified and sub-cultured in an isolation chamber with a laminar flow.

\section{Evaluation of different agrochemicals in in vitro condition}

Different concentrations of fungicides, herbicides, fertilizers were tested for the growth inhibition and sclerotial formation of M. phaseolina using poisoned food technique (Sinclair \& Dhingra, 1985). The required quantity of each chemical was incorporated aseptically in $100 \mathrm{ml}$ of PDA in $250 \mathrm{ml}$ flasks to make various concentrations of fungicides (50, 100, 250, 500, 1000, 1500 and 2000 ppm), herbicides $(500,1000,1500,2000 \mathrm{ppm})$ and fertilizers $(1000,2000,3000$ and $4000 \mathrm{ppm}$ ). The medium was shaken well to give uniform dispersal of the chemical and then $20 \mathrm{ml}$ of medium was poured aseptically to each plate with four replications. After solidification, the plates were inoculated with mycelial discs of $4 \mathrm{~mm}$ diameter of five days old culture. The mycelium disc which was placed in the center of the plates, in an inverted position to make a direct contact with the poisoned medium and was incubated at 28 $\pm 1{ }^{0} \mathrm{C}$ for seven days.

The linear growth of the fungal colonies was measured from two different angels in millimeter $(\mathrm{mm})$ and the average values were calculated. Sclerotial formations were counted in fungal culture suspensions under the microscope at low power (10X). The fungal culture suspension was prepared by vigorously shaking the $4 \mathrm{~mm}$ mycelial disc of the fungus in $10 \mathrm{ml}$ sterilized distilled water. The relative degree of formation of sclerotia was recorded as below. 


\begin{tabular}{|l|l|l|}
\hline $\begin{array}{l}\text { No. of sclerotia } \\
\text { per microscopic } \\
\text { field (10X) }\end{array}$ & Grade & Sign \\
\hline $\mathbf{0}$ & Absent & - \\
$\mathbf{1 - 4}$ & Scanty & + \\
$\mathbf{5 - 8}$ & Moderate & ++ \\
$\mathbf{9 - 1 5}$ & Good & +++ \\
$\mathbf{> 1 5}$ & Abundant & ++++ \\
\hline
\end{tabular}

The per cent inhibition of growth of the fungus in each treatment was calculated by using the following formula described by Vincent (1947).

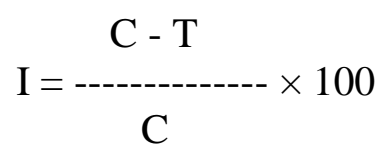

Where,

$\mathrm{I}=$ Per cent inhibition

$\mathrm{C}=$ Colony diameter in control $(\mathrm{mm})$

$\mathrm{T}=$ Colony diameter in respective treatment

\section{Results and Discussion}

Effects of different non systemic fungicides on the growth and sclerotial formation of M. phaseolina

The relative efficacy of seven different nonsystemic fungicides was tested at 500, 1000, 1500 and 2000 ppm concentrations. The observations regarding per cent inhibition of linear growth and sclerotial formation are presented in Table 1. The data presented in Table 1 revealed that zineb, gave cent per cent inhibition of mycelium growth and sclerotial formation at minimum concentration of 500 ppm. Mancozeb and copper hydroxide at $2000 \mathrm{ppm}$ concentration were quite effective and gave 88.89 and 83.35 per cent mean growth inhibition. Thiram was found moderately effective with per cent inhibition of 58.08 per cent whereas wettable sulphur, chlorothalonil and copper oxychloride were less effective among all nonsystemic fungicides tested. The maximum toxicity index (400) was observed in zineb. The growth inhibition and sclerotial formation was decreased with the increase in concentrations for all the chemicals tested.

Zineb do not allowed sclerotial formation even at lower concentration of $500 \mathrm{ppm}$. Thiram and mancozeb at 1000 ppm concentration found effective and completely suppress the sclerotial formation. Wettable sulphur failed to restrict the sclerotial formation. These results agreement with Syed and Ghaffar (1995) found that benomyl, mancozeb, zineb and captan showed significant protection of root rot caused by $R$. bataticola in soybean.

Effects of different systemic fungicides on the growth and sclerotial formation of $M$. Phaseolina

The relative efficacy of seven different systemic fungicides was tested at 50, 100, 250 and $500 \mathrm{ppm}$ concentrations. The observations regarding per cent inhibition of linear growth and sclerotial formation are presented in Table 2. The analysis of data presented in Table 2 revealed that all systemic fungicides were capable of inhibiting the growth at various concentrations as compared to control. All the fungicide gave more than 50 per cent growth inhibition of test fungus at lower concentration of $50 \mathrm{ppm}$ except carboxin and hexaconazole. Carbendezim at 500 ppm gave cent per cent inhibition of pathogen with mean inhibition of $97.81 \%$. Thiophanate methyl and tebuconazole were also found effective with mean inhibition of 84.57 and 80.50 per cent, respectively. Difenconoazole, carboxin, hexaconazole and tridemorph were moderately effective with mean inhibition of 75.76, 62.55, 61.55 and 61.24 per cent, respectively. 
Table.1 Mean per cent inhibition of $M$. phaseolina at different conc. of various non systemic fungicides (7 day after inoculation)

\begin{tabular}{|c|c|c|c|c|c|c|}
\hline \multirow[b]{2}{*}{ Fungicide } & \multicolumn{4}{|c|}{ Conc. (ppm) per cent inhibition* } & \multirow[t]{2}{*}{ Mean } & \multirow{2}{*}{$\begin{array}{l}\text { Toxicity } \\
\text { Index }\end{array}$} \\
\hline & 500 & 1000 & 1500 & 2000 & & \\
\hline Zineb & $\begin{array}{c}\text { A } 100.0 \\
\text { B - }\end{array}$ & $\begin{array}{c}100.00 \\
--\end{array}$ & $\begin{array}{c}100.00 \\
--\end{array}$ & $\begin{array}{c}100.00 \\
--\end{array}$ & 100.00 & 400 \\
\hline Copper hydroxide & $\begin{array}{c}\text { A } 17.78 \\
\text { B ++ }\end{array}$ & $\begin{array}{c}51.15 \\
+\end{array}$ & $\begin{array}{c}62.25 \\
+\end{array}$ & $\begin{array}{c}83.35 \\
--\end{array}$ & 53.63 & 214.52 \\
\hline Thiram & $\begin{array}{c}\text { A } 48.68 \\
\text { B }+\end{array}$ & $\begin{array}{c}53.61 \\
--\end{array}$ & $\begin{array}{c}63.36 \\
--\end{array}$ & $\begin{array}{c}66.68 \\
--\end{array}$ & 58.08 & 232.32 \\
\hline Copper oxychloride & $\begin{array}{c}\text { A } 22.25 \\
\text { B ++ }\end{array}$ & $\begin{array}{c}36.68 \\
++\end{array}$ & $\begin{array}{c}38.89 \\
+\end{array}$ & $\begin{array}{c}42.24 \\
--\end{array}$ & 35.01 & 140.04 \\
\hline Chlorothalonil & $\begin{array}{c}\text { A } 4.46 \\
\text { B ++ }\end{array}$ & $\begin{array}{c}23.36 \\
++\end{array}$ & $\begin{array}{c}27.78 \\
++\end{array}$ & $\begin{array}{c}67.78 \\
+\end{array}$ & 30.85 & 123.4 \\
\hline Mancozeb & $\begin{array}{c}\text { A } 2.25 \\
\text { B }++++\end{array}$ & $\begin{array}{c}50.00 \\
--\end{array}$ & $\begin{array}{c}57.78 \\
--\end{array}$ & $\begin{array}{c}88.89 \\
--\end{array}$ & 49.74 & 198.96 \\
\hline Wettable sulphur & $\begin{array}{l}\text { A } 2.36 \\
\text { B +++ }\end{array}$ & $\begin{array}{l}2.90 \\
+++\end{array}$ & $\begin{array}{l}3.35 \\
+++\end{array}$ & $\begin{array}{c}44.46 \\
++\end{array}$ & 13.26 & 53.04 \\
\hline Control & 0.00 & 0.00 & 0.00 & 0.00 & 0.00 & 0.00 \\
\hline & \multicolumn{2}{|c|}{ Fungicide (F) } & \multicolumn{3}{|c|}{ Concentration $(\mathrm{C})$} & $\mathrm{F} \times \mathrm{C}$ \\
\hline S.Em. \pm & \multicolumn{2}{|c|}{0.027} & \multicolumn{3}{|c|}{0.0357} & 0.0714 \\
\hline C.D. at 5\% & \multicolumn{2}{|c|}{0.0761} & \multicolumn{3}{|c|}{0.1006} & 0.2011 \\
\hline
\end{tabular}

$*$ Mean of four replications \# Maximum toxicity index $=400.00$

$\mathrm{A}=$ Growth inhibition $\mathrm{B}=$ Sclerotial formation:

++++ = abundant; +++ = good; ++ = moderate; + = scanty; --= absent

Table.2 Mean per cent inhibition of growth and sclerotial formation of M. phaseolina at different concentrations of various systemic fungicides after seven days of incubation at $28 \pm 1{ }^{\circ} \mathrm{C}$

\begin{tabular}{|c|c|c|c|c|c|c|}
\hline \multirow[t]{2}{*}{ Fungicide } & \multicolumn{4}{|c|}{ Conc. $(\mathrm{ppm}) /$ per cent inhibition* } & \multirow[t]{2}{*}{ Mean } & \multirow{2}{*}{$\begin{array}{l}\text { Toxicity } \\
\text { Index }\end{array}$} \\
\hline & 50 & 100 & 250 & 500 & & \\
\hline Tridemorph 25 EC & $\begin{array}{l}\text { A } 55.55 \\
\text { B }++\end{array}$ & $\begin{array}{c}61.72 \\
++\end{array}$ & $\begin{array}{c}61.12 \\
--\end{array}$ & $\begin{array}{c}66.67 \\
--\end{array}$ & 61.24 & 245.16 \\
\hline Difenoconazole25 EC & $\begin{array}{l}\text { A } 72.23 \\
\text { B }++\end{array}$ & $\begin{array}{c}74.45 \\
++\end{array}$ & $\begin{array}{c}77.78 \\
++\end{array}$ & $\begin{array}{c}77.78 \\
++\end{array}$ & 75.56 & 302.24 \\
\hline Carboxin 75 wp & $\begin{array}{c}\text { A } 38.88 \\
\text { B }++\end{array}$ & $\begin{array}{c}50.22 \\
++\end{array}$ & $\begin{array}{l}77.78 \\
+\end{array}$ & $\begin{array}{c}83.34 \\
--\end{array}$ & 62.55 & 250.20 \\
\hline $\begin{array}{l}\text { Thioaphanate methyl } \\
\text { 70wp }\end{array}$ & $\begin{array}{c}\text { A } 83.34 \\
\text { B -- }\end{array}$ & $\begin{array}{c}84.45 \\
--\end{array}$ & $\begin{array}{c}84.95 \\
--\end{array}$ & $\begin{array}{c}85.55 \\
--\end{array}$ & 84.57 & 338.29 \\
\hline Carbendazim 50 wp & $\begin{array}{l}\text { A } 94.30 \\
\text { B -- }\end{array}$ & $\begin{array}{c}97.71 \\
--\end{array}$ & $\begin{array}{c}99.22 \\
--\end{array}$ & $\begin{array}{c}100.00 \\
--\end{array}$ & 97.81 & 391.24 \\
\hline Hexaconazole 5 EC & $\begin{array}{c}\text { A } 33.78 \\
\text { B -- }\end{array}$ & $\begin{array}{c}66.30 \\
--\end{array}$ & $\begin{array}{c}70.98 \\
--\end{array}$ & $\begin{array}{l}75.56 \\
--\end{array}$ & 61.55 & 246.62 \\
\hline Tebuconazole 25.9 EC & $\begin{array}{c}\text { A } 77.39 \\
\text { B }++\end{array}$ & $\begin{array}{c}79.22 \\
++\end{array}$ & $\begin{array}{c}80.08 \\
++\end{array}$ & $\begin{array}{c}85.31 \\
+\end{array}$ & 80.50 & 322.32 \\
\hline Control & 0.00 & 0.00 & 0.00 & 0.00 & 0.00 & 0.00 \\
\hline & \multicolumn{2}{|c|}{ Fungicide $(\mathrm{F})$} & \multicolumn{3}{|c|}{ Concentration $(\mathrm{C})$} & $\mathrm{F} \times \mathrm{C}$ \\
\hline S.Em. \pm & \multicolumn{2}{|c|}{0.0653} & \multicolumn{3}{|c|}{0.0864} & 0.1728 \\
\hline C.D. at $5 \%$ & \multicolumn{2}{|c|}{0.184} & \multicolumn{3}{|c|}{0.2434} & 0.4868 \\
\hline
\end{tabular}

* Mean of four replications \# Maximum toxicity index $=400.00$

A = Growth inhibition $\mathrm{B}=$ Sclerotial formation:

++++ = abundant; +++ = good; ++ = moderate; + = scanty; --=absent 
Table.3 Mean per cent inhibition of $M$. phaseolina at different concentrations of various combination of fungicide after seven days of incubation at $28 \pm 1{ }^{\circ} \mathrm{C}$

\begin{tabular}{|c|c|c|c|c|c|c|}
\hline \multirow[t]{2}{*}{ Fungicide } & \multicolumn{4}{|c|}{ Conc. $(p p m) /$ per cent inhibition* } & \multirow[t]{2}{*}{ Mean* } & \multirow[t]{2}{*}{ Toxicity Index } \\
\hline & 250 & 500 & 1000 & 2000 & & \\
\hline Iprodione 25\% WP + Carbendazim 25\% WP & $\begin{array}{l}\text { A } 26.6 \\
\text { В }+++\end{array}$ & $\begin{array}{c}40.24 \\
+++\end{array}$ & $\begin{array}{c}47.15 \\
++\end{array}$ & $\begin{array}{c}87.25 \\
++\end{array}$ & 50.30 & 201.2 \\
\hline $\begin{array}{l}\text { Carbendazim 12\% WP + } \\
\text { Mancozeb } 63 \% \text { WP }\end{array}$ & $\begin{array}{c}\text { A } 72.4 \\
\text { B }+\end{array}$ & $\begin{array}{c}77.13 \\
+\end{array}$ & $\begin{array}{c}100.0 \\
--\end{array}$ & $\begin{array}{c}100.0 \\
--\end{array}$ & 87.47 & 349.88 \\
\hline Cymoxanil 8\% WP + Mancozeb 64\% WP & $\begin{array}{c}\text { A } 66.64 \\
\text { B }+\end{array}$ & $\begin{array}{c}83.35 \\
+\end{array}$ & $\begin{array}{c}100.0 \\
--\end{array}$ & $\begin{array}{c}100.0 \\
--\end{array}$ & 87.50 & 350.00 \\
\hline Metiram 55\%WP + Pyraclostrobin 5\% WG & $\begin{array}{c}\text { A } 78.56 \\
\text { B }+\end{array}$ & $\begin{array}{l}83.96 \\
+\end{array}$ & $\begin{array}{c}100.0 \\
--\end{array}$ & $\begin{array}{c}100.0 \\
--\end{array}$ & 90.63 & 362.52 \\
\hline Zineb 60\% WP + Hexaconazole 4\% WP & $\begin{array}{c}\text { A } 87.56 \\
\text { B }+\end{array}$ & $\begin{array}{c}88.85 \\
+\end{array}$ & $\begin{array}{c}100.0 \\
--\end{array}$ & $\begin{array}{c}100.0 \\
--\end{array}$ & 94.10 & 376.4 \\
\hline Pyraclostrobin 13.3\% WP + Epoxyconazol 5\% WP & $\begin{array}{l}\text { A } 100 \\
\text { B -- }\end{array}$ & $\begin{array}{c}100.0 \\
--\end{array}$ & $\begin{array}{c}100.0 \\
--\end{array}$ & $\begin{array}{c}100.0 \\
--\end{array}$ & 100.0 & 400.00 \\
\hline Metalaxyl 8\% WP + Mancozeb 64\% WP & $\begin{array}{l}\text { A100.0 } \\
\text { B -- }\end{array}$ & $\begin{array}{c}100.0 \\
--\end{array}$ & $\begin{array}{c}100.0 \\
--\end{array}$ & $\begin{array}{c}100.0 \\
--\end{array}$ & 100.0 & 400.00 \\
\hline Control & 0.00 & 0.00 & 0.00 & 0.00 & 0.00 & 0.00 \\
\hline & \multicolumn{2}{|c|}{ Fungicide $(\mathrm{F})$} & \multicolumn{3}{|c|}{ Concentration $(\mathrm{C})$} & $\mathrm{F} \times \mathrm{C}$ \\
\hline S.Em. \pm & \multicolumn{2}{|c|}{0.0185} & \multicolumn{3}{|c|}{0.0245} & 0.049 \\
\hline C.D. at $5 \%$ & \multicolumn{2}{|c|}{0.0523} & \multicolumn{3}{|c|}{0.0692} & 0.1384 \\
\hline
\end{tabular}

* Mean of four replications \# Maximum toxicity index $=400.00$

$\mathrm{A}=$ Growth inhibition $\mathrm{B}=$ Sclerotial formation:

++++ = abundant ++++ good $;++=$ moderate $;+=$ scanty; $--=$ absent

Table.4 Mean per cent inhibition of $M$. phaseolina at different concentrations of various herbicides after seven days of incubation at $28 \pm 1{ }^{\circ} \mathrm{C}$

\begin{tabular}{|c|c|c|c|c|c|c|}
\hline \multirow[t]{2}{*}{ Herbicide } & \multicolumn{4}{|c|}{ Conc. $(\mathrm{ppm}) /$ per cent inhibition* } & \multirow[t]{2}{*}{ Mean } & \multirow[t]{2}{*}{ Toxicity Index } \\
\hline & 500 & 1000 & 1500 & 2000 & & \\
\hline Propaquizafop $10 \%$ EC & $\begin{array}{c}\text { A } 86.51 \\
\text { B }+\end{array}$ & $\begin{array}{c}97.71 \\
--\end{array}$ & $\begin{array}{c}100.00 \\
--\end{array}$ & $\begin{array}{c}100.00 \\
--\end{array}$ & 96.05 & 384.20 \\
\hline Quizalophop-p-ethyle 5\% EC & $\begin{array}{c}\text { A } 100.0 \\
\text { B -- }\end{array}$ & $\begin{array}{c}100.00 \\
--\end{array}$ & $\begin{array}{c}100.00 \\
--\end{array}$ & $\begin{array}{c}100.00 \\
--\end{array}$ & 100.00 & 400.00 \\
\hline Pendimethalin $30 \%$ EC & $\begin{array}{c}\text { A } 16.68 \\
\text { B ++ }\end{array}$ & $\begin{array}{c}22.25 \\
++\end{array}$ & $\begin{array}{c}38.85 \\
++\end{array}$ & $\begin{array}{c}55.58 \\
++\end{array}$ & 33.34 & 121.36 \\
\hline Metasulfuron-methyl 20\% WG & $\begin{array}{c}\text { A } 48.88 \\
\text { B + }\end{array}$ & $\begin{array}{c}57.78 \\
+\end{array}$ & $\begin{array}{c}62.24 \\
+\end{array}$ & $\begin{array}{c}66.25 \\
+\end{array}$ & 58.78 & 235.12 \\
\hline Glyphosat $41 \%$ EC & $\begin{array}{l}\text { A } 8.77 \\
\text { B +++ }\end{array}$ & $\begin{array}{c}18.88 \\
+++\end{array}$ & $\begin{array}{c}27.77 \\
++\end{array}$ & $\begin{array}{c}42.26 \\
+\end{array}$ & 24.42 & 97.68 \\
\hline Oxadiargyl 6\% EC & $\begin{array}{c}\text { A } 61.13 \\
\text { B }+\end{array}$ & $\begin{array}{c}73.35 \\
--\end{array}$ & $\begin{array}{c}94.46 \\
--\end{array}$ & $\begin{array}{c}100.00 \\
--\end{array}$ & 82.23 & 328.92 \\
\hline Oxyfluorfen $23.5 \%$ EC & $\begin{array}{c}\text { A } 75.57 \\
\text { B + }\end{array}$ & $\begin{array}{c}85.57 \\
--\end{array}$ & $\begin{array}{c}93.68 \\
--\end{array}$ & $\begin{array}{c}100.00 \\
--\end{array}$ & 88.70 & 354.80 \\
\hline Fenoxaprop-p-ethyle $10 \%$ EC & $\begin{array}{c}\text { A } 4.24 \\
\text { B ++ }\end{array}$ & $\begin{array}{c}61.14 \\
++\end{array}$ & $\begin{array}{c}67.69 \\
++\end{array}$ & $\begin{array}{c}83.35 \\
++\end{array}$ & 54.10 & 216.40 \\
\hline Paraquate dichloride $24 \%$ SL & $\begin{array}{c}\text { A } 37.78 \\
\text { B ++ }\end{array}$ & $\begin{array}{c}77.77 \\
+\end{array}$ & $\begin{array}{c}75.77 \\
+\end{array}$ & $\begin{array}{c}88.84 \\
+\end{array}$ & 65.30 & 65.30 \\
\hline Control & 0.00 & 0.00 & 0.00 & 0.00 & 0.00 & 0.00 \\
\hline & \multicolumn{2}{|c|}{ Herbicide $(\mathrm{H})$} & \multicolumn{3}{|c|}{ Concentration $(\mathrm{C})$} & $\mathrm{H} \times \mathrm{C}$ \\
\hline S.Em. \pm & \multicolumn{2}{|c|}{1.0524} & \multicolumn{3}{|c|}{1.578} & 3.157 \\
\hline C.D. at 5\% & \multicolumn{2}{|c|}{2.952} & \multicolumn{3}{|c|}{4.429} & 8.858 \\
\hline
\end{tabular}

* Mean of four replications \# Maximum toxicity index $=400.00$

$\mathrm{A}=$ Growth inhibition $\mathrm{B}=$ Sclerotial formation:

++++ = abundant; +++ = good $+++=$ moderate $;+=$ scanty; $--=$ absent 
Table.5 Mean per cent inhibition of M. phaseolina at different concentrations various fertilizers after seven days of incubation at $28 \pm 1{ }^{\circ} \mathrm{C}$

\begin{tabular}{|c|c|c|c|c|c|c|}
\hline \multirow[t]{2}{*}{ Fertilizer } & \multicolumn{4}{|c|}{ Conc. $(\mathbf{p p m}) /$ per cent inhibition* } & \multirow[t]{2}{*}{ Mean } & \multirow{2}{*}{$\begin{array}{l}\text { Toxicity } \\
\text { Index }\end{array}$} \\
\hline & 1000 & 2000 & 3000 & 4000 & & \\
\hline Diammonium phosphate & A100.0B -- & $\begin{array}{c}100.0 \\
--\end{array}$ & $\begin{array}{c}100.0 \\
--\end{array}$ & $\begin{array}{c}100.0 \\
--\end{array}$ & 100.0 & 400.0 \\
\hline Ammonium sulphate & $\begin{array}{c}\text { A } 67.6 \\
\text { B }+\end{array}$ & $\begin{array}{c}69.9 \\
+\end{array}$ & $\begin{array}{c}74.6 \\
+\end{array}$ & $\begin{array}{c}76.6 \\
--\end{array}$ & 72.2 & 288.7 \\
\hline Urea & $\begin{array}{c}\text { A } 61.3 \\
\text { B }+\end{array}$ & $\begin{array}{c}65.3 \\
+\end{array}$ & $\begin{array}{c}72.9 \\
+\end{array}$ & $\begin{array}{c}74.9 \\
--\end{array}$ & 68.6 & 274.4 \\
\hline Single Super Phosphate & $\begin{array}{c}\text { A } 54.3 \\
\text { B }++\end{array}$ & $\begin{array}{c}64.3 \\
++\end{array}$ & $\begin{array}{c}71.7 \\
+\end{array}$ & $\begin{array}{c}72.1 \\
+\end{array}$ & 65.6 & 262.4 \\
\hline Murate of potash & $\begin{array}{l}\text { A } 21.1 \\
\text { B }+++\end{array}$ & $\begin{array}{l}32.2 \\
+++\end{array}$ & $\begin{array}{c}53.1 \\
++\end{array}$ & $\begin{array}{c}64.6 \\
+\end{array}$ & 42.8 & 171.0 \\
\hline $\begin{array}{l}\text { Narmadaphos } \quad \text { CAN } \\
(20: 20: 0)\end{array}$ & $\begin{array}{l}\text { A } 24.9 \\
\text { B }+++\end{array}$ & $\begin{array}{l}34.3 \\
+++\end{array}$ & $\begin{array}{l}42.6 \\
+++\end{array}$ & $\begin{array}{c}52.5 \\
++\end{array}$ & 38.6 & 154.3 \\
\hline IFFCON:P:K (12:32:16) & $\begin{array}{l}\text { A } 21.9 \\
\text { B }+++\end{array}$ & $\begin{array}{l}35.9 \\
+++\end{array}$ & $\begin{array}{l}42.9 \\
+++\end{array}$ & $\begin{array}{l}47.5 \\
+++\end{array}$ & 37.1 & 148.4 \\
\hline Control & 0.00 & 0.00 & 0.00 & 0.00 & 0.00 & 0.00 \\
\hline & \multicolumn{2}{|c|}{ Fungicide (F) } & \multicolumn{3}{|c|}{ Concentration $(\mathrm{C})$} & $\mathrm{F} \times \mathrm{C}$ \\
\hline S.Em. \pm & \multicolumn{2}{|c|}{0.37} & \multicolumn{3}{|c|}{0.75} & 0.2775 \\
\hline C.D. at $5 \%$ & \multicolumn{2}{|c|}{1.05} & \multicolumn{3}{|c|}{2.10} & 2.205 \\
\hline
\end{tabular}

* Mean of four replications \# Maximum toxicity index $=400.00$

$\mathrm{A}=$ Growth inhibition $\mathrm{B}=$ Sclerotial formation:

$++++=$ abundant $;+++=$ good $;++=$ moderate $;+=$ scanty; $--=$ absent

Maximum toxicity index of 391.24, 338.29 and 322.32 was observed in carbendazim, thioaphanate methyl and tebuconazole fungicides whereas minimum toxicity 245.16 was observed in tridemorph. The effect of different concentrations of systemic fungicides on sclerotial formation was found correlated with the inhibition of growth. No sclerotial formation was observed in all concentrations of carbendazim, thiphanate methyl and hexaconazole.

Good sclerotial formation was observed in tebuconazole and difenoconazole. Tridemorph and carboxin supported moderate to scanty sclerotial formation. Similar results found in Singh (1997) recorded carbendazim, thiophanate methyl and mancozeb $0.2 \%$ as most effective growth inhibitor of $M$. phaseolina in in vitro.
Effects of different combination of fungicides on the growth and sclerotial formation of $M$. phaseolina

Efficacy of seven different combinations of fungicide tested against pathogen $M$. phaseolina at 250, 500, 1000 and 2000 ppm concentration. The observation regarding per cent growth inhibition and sclerotial formation are presented in Table 3.

It is inferred from the data presented in Table 3 that all the combination of fungicides were found effective per cent growth mean inhibition of fungus. The pyraclostrobin 13.3\% WP + epoxyconazole 5\% WP and metalaxyl $8 \%$ + mancozeb $64 \%$ gave cent per cent mycelium inhibition and no sclerotial formation was observed at all their concentration tested. 
The cymoxanil- $8 \%+$ mancozeb $64 \%$, carbendazim $12 \%+$ mancozeb $63 \%$, metiram $55 \% \mathrm{WP}+$ pyraclostrobin $5 \% \mathrm{WG}$ and zineb $60 \% \mathrm{WP}+$ hexaconazole $4 \% \mathrm{WP}$ were found effective and gave cent per cent mycelium inhibition at higher concentration of 1000 and $2000 \mathrm{ppm}$ and completely suppress the sclerotial formation. Iprodione $25 \%+$ carbendazim $25 \%$ gave minimum mycelium inhibition and good sclerotial formation. Maximum toxicity index of 400 was recorded in pyraclostrobin $13.3 \% \mathrm{WP}+$ epoxyconazole 5\% WP and metalaxyl 8\% + mancozeb 64\%.

Effects of different herbicides on the growth and sclerotial formation of $M$. Phaseolina

Efficacy of nine different herbicides was tested against $M$. phaseolina at 250, 500, 1000 and $2000 \mathrm{ppm}$ concentration. The observation regarding per cent growth inhibition and sclerotial formation are presented in Table 4.

The data presented in Table 4 revealed that all herbicides were significantly superior in inhibiting the growth of the test fungus at different concentrations as compared to the control (Table 4). Among them, quizalophopp-ethyle 5\% EC gave cent per cent inhibition of mycelial growth at all concentration tested followed by propaquizafop 10\% EC which gave cent per cent growth inhibition at 1500 and $2000 \mathrm{ppm}$. Oxadiargyl 6\% EC and oxyfluorfen $23.5 \%$ EC were also found effective and gave complete inhibition of mycelial growth at higher concentration of 2000 ppm. Paraquate dichloride, metasulfuron-methyl, fenoxaprop-p-ethyle were moderately effective with mean growth inhibition of $65.30,58.78,54.10$ per cent. Whereas pendimethalin 30\% EC, glyphosate $41 \%$ EC were performed poor as compared to other herbicides. The formation of sclerotial was completely absent in quizalophop-pethyle $5 \%$ EC, propaquizafop $10 \%$ EC, oxadiargy 6\% EC and oxyfluorfen 23.5 EC at
$1000 \mathrm{ppm}$ concentration whereas, good to moderate sclerotial formation was observed in rest of herbicides tested. Maximum toxicity index of 400 was recorded in quizalophop-pethyle. Similar results found by De et al., (2007) in which quizalofop-ethyl exhibited 100 per cent inhibition of growth of $M$. phaseolina at $25 \mathrm{micro} \mathrm{g} / \mathrm{ml}$ after $24 \mathrm{hrs}$.

Effects of various fertilizers on the growth and sclerotial formation of the $M$. Phaseolina

Relative efficacy of seven different fertilizers in inhibiting the fungal growth was evaluated using poisoned food technique.

Per cent growth inhibition and sclerotial formation recorded for various fertilizers are presented in Table 5 growth of fungus as compared to control. Among them, diammonium phosphate was most effectively and gave cent per cent growth inhibition and no sclerotial formation was recorded at all concentration. Ammonium sulphate, urea and SSP at $3000 \mathrm{ppm}$ to $4000 \mathrm{ppm}$ gave more than 70.0 per cent growth inhibition and supported moderately sclerotial formation. Murate of potash, Narmada CAN (20:20:0) and IFFCON:P:K (12:32:16) were moderately effective and gave $42.80,38.60$ and 37.10 per cent growth inhibition and supported good to moderate sclerotial formation. The toxicity index of 400 was recorded in treatment of diammonium phosphate on the basis of maximum toxicity index of 400. Present result agreement with Khalid-Iftikhar et al., (2001) conducted experiment in Punjab, Pakistan and results showed that $\mathrm{N}, \mathrm{P}$ and $\mathrm{K}$ at higher amounts were more effective in reducing the dry root rot disease

In conclusions all the seven non systemic fungicides tested Zineb gave cent per cent inhibition of mycelial growth and sclerotial formation at minimum concentration of 500 ppm. 
The most of systemic fungicides were capable of inhibiting the growth as compared to control. Carbendezim at 500 ppm gave cent per cent inhibition of pathogen.

Efficacy different combinations of fungicide tested against $M$. phaseolina and they gave more than 50 per cent growth inhibition of test fungus. The pyraclostrobin $13.3 \% \mathrm{WP}+$ epoxyconazole 5\% WP and metalaxyl $8 \%+$ mancozeb $64 \%$ completely inhibited mycelium and sclerotial formation.

Among various herbicides, most of herbicides were effective in inhibiting the growth of the test fungus as compared to the control. Quizalophop-p-ethyle 5\% EC, propaquizafop $10 \%$ EC which also gave cent per cent growth inhibition at 1500 and 2000 ppm. Oxadiargyl $6 \%$ EC and oxyfluorfen $23.5 \%$ EC were also found effective and gave complete inhibition of mycelial growth at higher concentration of 2000 ppm.

The chemical fertilizers were found to reduce the growth of fungus as compared to control. Diammonium phosphate was found quite effective completely restricted mycelial growth and sclerotial formation.

\section{References}

Anonymous, 2017: Area, production and productivity of soybean crop. State Agril. Department of Maharashtra.

Anahosur, K. H., Patil, S. H. and Hegde, R. K. 1984. Effect of herbicides on Macrophomina phaseolina (Tassi) Goid. Causing charcoal rot of sorghum. Pesticides. 18(4): 11-12.
Devi. P. T., and Singh, R. H. 1997. Screening of fungicides against seedling mortality of blackgram caused by Macrophomina phaseolina. Legume Res. 20 (2): 71-76.

Khalid Iftikhar Ilyas, Riaz Ahmad, M. B. and Iqbal, M. A. 2001. Effect of inorganic $(\mathrm{N}, \mathrm{P}, \mathrm{K})$ soil amendments on dry root rot disease of chickpea. Pakistan J. Phytopathology. 13(2): 116-120.

Muthusamy, S., and Mariappan, V. 1991. Disintegration of sclerotia of Macrophomina phaseolina (soybean isolate) by oil cake extracts. Indian Phytopath. 44: 271-273.

Singh, C. 1997. Modern techniques of raising field crop. Oxford and IBH Pub Co Pvt Ltd New Delhi 523 pp.

Srikant, K., Ramamurthy, R., Yashoda H., Kulkarni, S. and Hegde, Y. 1992. In vitro evaluations of herbicides against root rot of cotton. Pesticide Res J. 19 (2): 149-151.

Syed, B., Hque and Abdul Ghaffar. 1995. Effect of Bradyrhizobium japanicum and fungicides in the control of root rot disease of soybean. Pakistan J Bot. 27(1): 227-232.

Vincent, J. M. 1927. Distoration of fungal hyphae in presence of certain inhabitors. Nature 15:850.

Yang, X. B. and Navi, S. S. 2005. First report of charcoal rot epidemics caused by Macrophomina phaseolina in soybean, in IOWA. Pl Dis 89(5): 626.

Zafar Iqbal. 2012. Soybean Diseases and Their Management. Pub by Lambert Academic 88 p p.

\section{How to cite this article:}

Ingole, B. M. and Zade, S. B. 2020. Bioassay of Different Agrochemicals and Fertilizers against Root rot (Macrophomina phaseolina) of Soybean in in vitro. Int.J.Curr.Microbiol.App.Sci. 9(04): 582-589. doi: https://doi.org/10.20546/ijcmas.2020.904.071 